\title{
Recurrent sialoliths after excision of the bilateral submandibular glands for sialolithiasis treatment: A case report
}

\author{
XIAOXIA YING, JIANAN KANG, FUYIN ZHANG and HUI DONG \\ Department of Stomatology, The First Affiliated Hospital of Dalian Medical University, Dalian, Liaoning 116011, P.R. China
}

Received October 15, 2014; Accepted September 1, 2015

DOI: $10.3892 /$ etm.2015.2849

\begin{abstract}
Sialolithiasis is a common disease that is characterized by the obstruction of the salivary gland. Sialolithiasis mainly affects the submandibular glands and the Wharton's duct. However, bilateral sialolithiasis is a rare condition. In addition, recurrence of sialoliths subsequent to surgical excision of the submandibular gland for the treatment of sialolithiasis has been rarely reported. The present study reported a case presenting with recurrent sialoliths with sialadenitis in the residual Wharton's duct following the excision of bilateral submandibular glands. An 81-year-old man presented with a solid and painful mass in the left submandibular area. The patient had a history of bilateral submandibular sialolithiasis, and had undergone excision of bilateral submandibular glands with the right Wharton's duct 4 years earlier. Computed tomography scans demonstrated two calculi in the residual Wharton's duct, which were surgically removed without any complications. The present study discussed the mechanisms underlying sialolith formation subsequent to the excision of submandibular glands.
\end{abstract}

\section{Introduction}

Sialolithiasis is one of the most common diseases of the salivary gland, accounting for $\sim 50 \%$ of the obstructive and inflammatory diseases of the major salivary glands (1). The most common symptoms associated with sialolithiasis are pain and swelling of the affected salivary gland. Sialolithiasis is most frequent in male patients aged between 30-60 years (2). Sialolithiasis affects the submandibular glands and Wharton's duct in $80-90 \%$ of cases $(3)$, and is mainly unilateral $(2,4)$. Bilateral sialolithiasis is a rare condition, accounting for $1-3 \%$ of cases (5-7). A sialolith must always be removed, since a long term obstruction of the salivary gland duct can lead to

Correspondence to: Dr Hui Dong, Department of Stomatology, The First Affiliated Hospital of Dalian Medical University, 222 Zhongshan Road, Dalian, Liaoning 116011, P.R. China

E-mail: donghui760413@hotmail.com

Key words: sialolithiasis, recurrence, excision, Wharton's duct inflammation and infection. Surgical treatments, including the incision of Warthon's duct and sialendoscopy, and non-surgical treatments, including oral analgesics and antibiotics, have been used for the removal of sialoliths (8-10). Sialolithectomy is the preferred method for the removal of the stone, since this treatment results in immediate relief of the pressure inside the gland by releasing the retained saliva. Excision of the affected salivary gland and its associated duct is also an option for the treatment of sialolithiasis, particularly in cases with recurrent stone formation. The present study reported the case of an 81-year-old man with two recurrent calculi and concurrent sialadenitis in the residual Wharton's duct, who had a history of excision of bilateral submandibular glands as a sialolithiasis treatment. The present study aimed to investigate the potential mechanisms underlying recurrent sialolith formation in residual Wharton's ducts following excision of the bilateral submandibular glands. Sialodochoplasty of the submandibular duct and careful removal of calculi may reduce the possibility of sialolith recurrence.

\section{Case report}

In February 2013, an 81-year-old man was admitted to the First Affiliated Hospital of Dalian Medical University (Dalian, China), complaining of a solid and painful mass in the left submandibular area. The pain and swelling had been present for $\sim 6$ months. The patient had presented repeated episodes of sialolithiasis in the right mandibular glands 4 years earlier. A previous computed tomography (CT) scan revealed bilateral salivary stones in the submandibular glands. The right stone was located in the Wharton's duct near the glandular hilum, and the left stone was located in the parenchyma of the gland (Fig. 1A). Excision of bilateral submandibular glands with the right Wharton's duct was performed in the First Affiliated Hospital of Dalian Medical University in November 2009. The patient had no history of alcohol consumption, smoking or drug abuse.

Clinical examination revealed normal intro-oral and extra-oral anatomical structures, with the exception of local indurated swelling in the left submandibular area. A CT scan of the floor of the mouth and the neck demonstrated the presence of two calcified masses below the inferior border of the mandible (Fig. 1B and C). The anterior smooth mass was adjacent to the location of the previously excised left Wharton's duct near the hilum of the gland, and the posterior irregular 

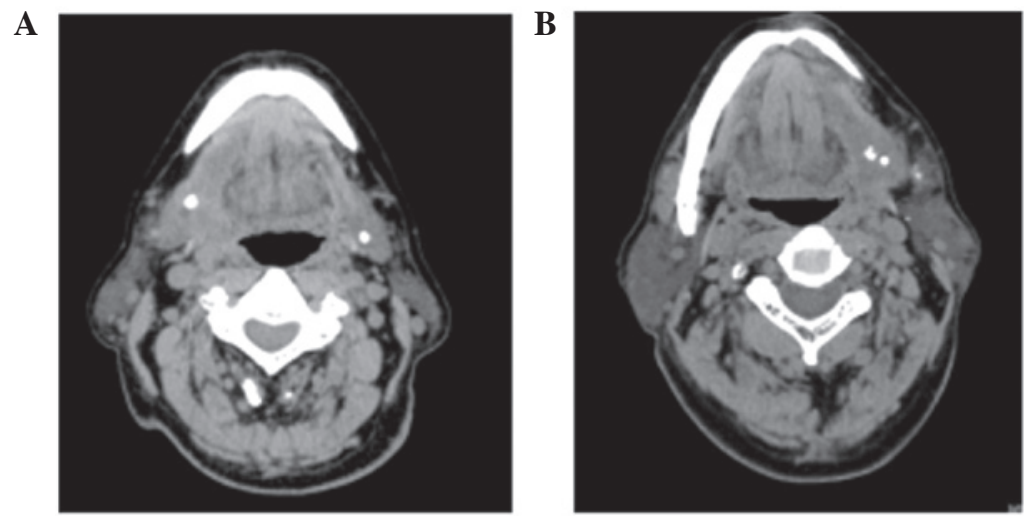

C

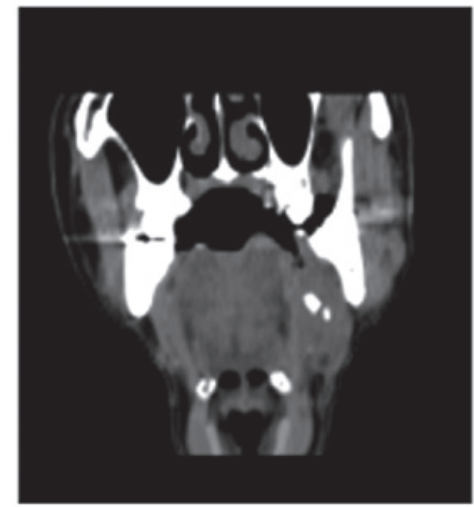

D

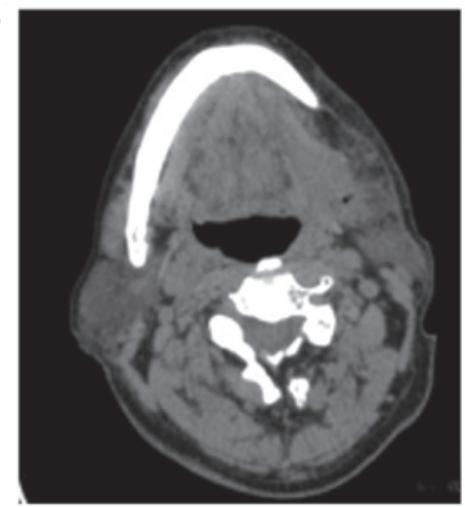

Figure 1. Computed tomography (CT) scans showing the detection of sialoliths. (A) Axial CT, performed in 2009 prior to the excision of bilateral salivary stones, demonstrated bilateral salivary stones in the submandibular glands. (B) Axial and (C) coronal CT scans performed in 2013 demonstrated two calcified masses below the inferior border of the mandible. (D) Postoperative CT performed in 2013 showed no radiopaque lesions of the floor of the mouth and the neck.

mass was in proximity to the anterior mass. Inflammation of the soft tissues around the radiopaque lesions was detected, and no radiographic evidence of residual left submandibular gland tissues was observed Based on the history of the patient and CT findings, the patient was diagnosed with recurrent sialolithiasis.

Subsequently, the calculi were surgically excised. Purulence was observed around the calculi, and thus incision and irrigation of the surrounding soft tissues were performed. Following surgery, the patient was treated with intravenous injection of $3 \mathrm{~g}$ cefazolin twice a day for 3 days. A postoperative CT scan showed no radiopaque lesions of the mouth floor and neck (Fig. 1D). The patient was discharged without any complications. The patient attended follow-up sessions every 6 months, and at the latest follow-up (May, 2014), the patient was disease-free.

\section{Discussion}

Sialolithiasis is the most common disease of the salivary glands, mainly affecting the submandibular glands (2). However, bilateral sialolithiasis is a rare condition accounting for $<3 \%$ of cases (6). Levy et al reported that bilateral sialolithiasis occurred in 4 out of 180 cases $(2.2 \%)$ with sialolithiasis (7). In addition, Tholen found that, in a cohort including 97 patients with sialolithiasis, only 1 patient (1\%) presented bilateral sialolithiasis (5). In the present case, the patient had a history of bilateral sialolithiasis, and had undergone excision of the bilateral submandibular glands and the right Wharton's duct. Recurrent sialolithiasis in the residual Wharton's duct occurred 4 years after the excision of the submandibular glands. Similarly, Markiewicz et al reported a case of recurrent sialolithiasis in a residual Wharton's duct 12 years after the excision of the submandibular glands (10). The case described in the present study along with that reported by Markiewicz et al (10) demonstrated signs of infection in the residual submandibular glands, suggesting that a long-term obstruction may lead to infection in a residual Wharton's duct.

The exact mechanisms underlying sialolith formation remain unclear. Several factors, such as abnormality in $\mathrm{Ca}^{2+}$ metabolism, altered $\mathrm{pH}$ of saliva and reduced salivary flow rate, have been suggested to contribute to sialolith formation (4). The high incidence of sialolith formation in the Wharton's duct may be due to the alkaline $\mathrm{pH}$ and high $\mathrm{Ca}^{2+}$ content of the saliva. In addition, a long ascending pathway of the Wharton's duct may contribute to reduced salivary flow rate due to gravity slowing the flow rate, as the saliva flows from the lower end to the upper end of the Wharton's duct, thus leading to sialolith formation (11). Furthermore, the salivary flow rate is further decreased subsequent to sialolith formation and infection, leading to aggravation of sialolithiasis.

The occurrence of sialoliths is rare, while the recurrence rate of sialolithiasis has been reported to be $\sim 8.9 \%$ (11). In the present case, recurrence of sialolithiasis occurred in the left Wharton's duct following excision of the bilateral submandibular glands. It is unlikely that the calculi were not detected during the time of the submandibular gland removal, since the Wharton's ducts were carefully examined and the right duct was removed due to the presence of calculi. Anatomically, a communication exists between the sublingual 
glandular complex and the submandibular gland duct. The sublingual gland communicates with the Wharton's duct via the Bartholin's duct or directly into the floor of the mouth (12). Therefore, the communication between the sublingual gland and the Wharton's duct is hypothesized to provide an anatomical basis for sialolith formation. In addition, the sublingual gland is predominantly a mucus secreting gland, and the viscous saliva in the relatively stagnant environment within the residual part of the Wharton's duct further facilitates sialolith formation $(10,13)$.

In conclusion, the present study described a rare case of recurrent sialoliths with concurrent sialadenitis in the residual Wharton's duct subsequent to excision of the bilateral submandibular glands for the treatment of sialolithiasis. Since the Wharton's duct can facilitate drainage of the sublingual gland, preservation of part of the Wharton's duct following excision of the mandibular gland for sialolithiasis is important in order to prevent sialadenitis of the sublingual bland. Sialodochoplasty of the duct of the submandibular gland in combination with careful massage of the duct from the bottom to the top to remove any undetected calculi may reduce the possibility of sialolith and sialadenitis recurrence in the sublingual gland.

\section{References}

1. Epker BN: Obstructive and inflammatory diseases of the major salivary glands. Oral Surg Oral Med Oral Pathol 33: 2-27, 1972.
2. Capaccio P, Torretta S, Ottavian F, Sambataro G and Pignataro L: Modern management of obstructive salivary diseases. Acta Otorhinolaryngol Ital 27: 161-172, 2007.

3. Bsoul SA, Flint DJ, Terezhalmy GT and Moore WS: Sialolithiasis. Quintessence Int 34: 316-317, 2003.

4. Haubrich J: Clinical aspects of non-tumorous diseases of the salivary glands. Arch Otorhinolaryngol 213: 1-59, 1976 (In German).

5. Tholen EF: Sialolithiasis. J Oral Surg (Chic) 7: 63-66, 1949.

6. Sunder VS, Chakravarthy C, Mikkilinine R and Mahoorkar S: Multiple bilateral submandibular gland sialolithiasis. Niger J Clin Pract 17: 115-118, 2014.

7. Levy DM, Remine WH and Devine KD: Salivary gland calculi. Pain, swelling associated with eating. JAMA 181: 1115-1119, 1962.

8. Juul ML and Wagner N: Objective and subjective outcome in 42 patients after treatment of sialolithiasis by transoral incision of Warthon's duct: A retrospective middle-term follow-up study. Eur Arch Otorhinolaryngol 271: 3059-3066, 2014.

9. Zenk J, Koch M, Klintworth N, König B, Konz K, Gillespie MB and Iro H: Sialendoscopy in the diagnosis and treatment of sialolithiasis: A study on more than 1000 patients. Otolaryngol Head Neck Surg 147: 858-863, 2012.

10. Markiewicz MR, Margarone JE III, Tapia JL and Aguirre A: Sialolithiasis in a residual Wharton's duct after excision of a submandibular salivary gland. J Laryngol Otol 121: 182-185, 2007.

11. Lustmann J, Regev E and Melamed Y: Sialolithiasis. A survey on 245 patients and a review of the literature. Int J Oral Maxillofac Surg 19: 135-138, 1990.

12. Zhang L, Xu H, Cai ZG, Mao C, Wang Y, Peng X, Zhu ZH and Yu GY: Clinical and anatomic study on the ducts of the submandibular and sublingual glands. J Oral Maxillofac Surg 68: 606-610, 2010.

13. Capaccio P, Torretta S, Ottavian F, Sambataro G and Pignataro L: Modern management of obstructive salivary diseases. Acta Otorhinolaryngol Ital 27: 161-172, 2007. 